\title{
Estimation of Support Reaction of Honeycomb Core Sandwich Panel from Deformation of Face Sheet: Influence of Fixing Method for Panel
}

\author{
Yoshinao Kishimoto $^{1 *}$, Yukiyoshi Kobayashi ${ }^{1}$, Toshihisa Ohtsuka ${ }^{1}$ and Kenichiro Nakamura ${ }^{1}$ \\ ${ }^{1}$ Department of Mechanical Engineering, Tokyo City University, 1-28-1 Tamazutsumi, Setagaya-ku, Tokyo, Japan \\ *ykishimo@tcu.ac.jp
}

Introduction. Honeycomb core sandwich panel is a composite structural material which consists of the honeycomb core and the face sheets. The panel has high specific strength, but the local compression makes a dent on the panel at relatively low load ${ }^{(1)}$. Although the distribution of the support reaction by the honeycomb core subjected to the local compression is still concerned, it is difficult to directly measure the support reaction by some measuring instruments such as strain gauges because the honeycomb core is crushed in the compression process. This study has developed a novel method to estimate the support reaction by the inverse analysis of the deformation of the face sheet ${ }^{(2)}$. The deformation of the face sheet is affected by the fixing method for the panel as well as the support reaction. In order to investigate the influence of the fixing method for the panel on the estimation accuracy of the proposed method, a series of the local compression tests was conducted with the bottom face fixed panel and the peripherally clamped panel.

Method. Figure 1 shows the experimental apparatus. The specimen is the honeycomb core sandwich panel made of A1100 aluminum alloy. The indenter applies the local compressive load on the panel. The laser displacement meter measures the deformation of the face sheet of the panel. Because the support reaction by the honeycomb core is the external load for the face sheet, the proposed method analyzes only the face sheet. Figure 2 shows the schematic of the mathematical model of the face sheet. The deformation of the face sheet $u$ is given as the boundary condition and the support reaction $f$ on the face sheet is estimated. In order to reduce the estimation error induced by the measurement error in the displacement $u$, the proposed method select the support reaction $f^{*}$ that minimizes the following evaluation function $\Pi$.

$$
\Pi=\left\|\boldsymbol{u}-A \boldsymbol{f}^{*}\right\|^{2}+\alpha\left\|\boldsymbol{f}^{*}-\boldsymbol{f}_{\mathbf{0}}\right\|^{2}
$$

Where the bold faces indicate the data vector of the displacement $u$ and the support reaction $f^{*}$. $A$ is the compliance matrix derived by the finite element method and $\alpha$ is the Tikhonov regularization parameter. $f_{0}$ is the reference solution.

Results. Figure 3 shows the estimation results and the experimental values of the support reaction of the panel. The experimental values were calculated by the crushing amount and the whole compression property of the honeycomb core. The whole compression property was measured in the preliminary experiment. As shown in Fig. 3, the estimation results have good agreement with

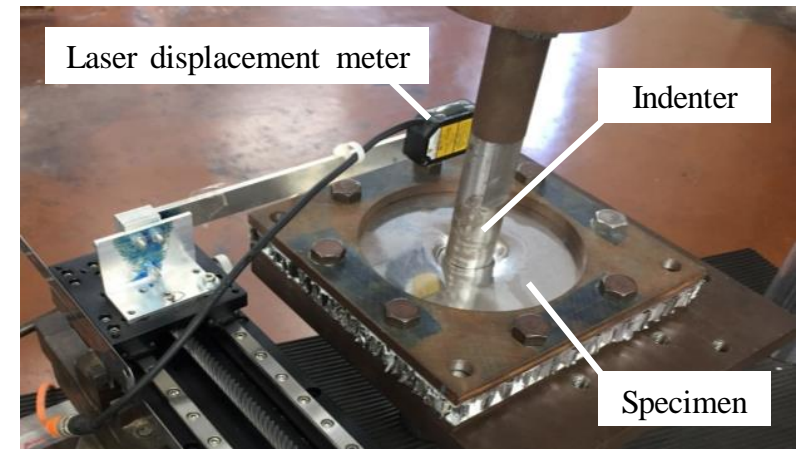

Fig. 1 Specimen and experimental apparatus

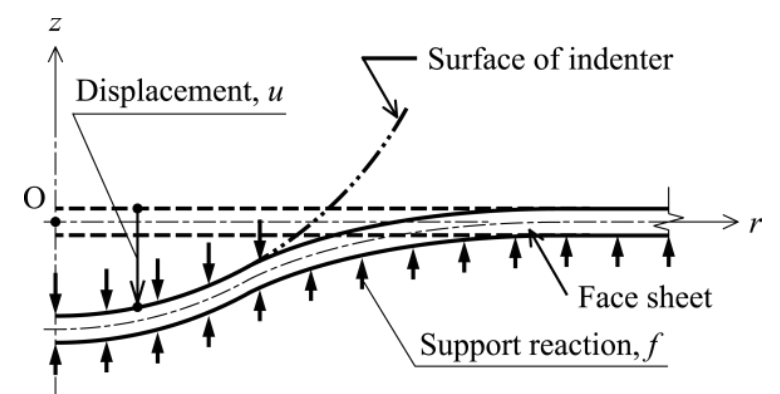

Fig. 2 Schematic of mathematical model of face sheet

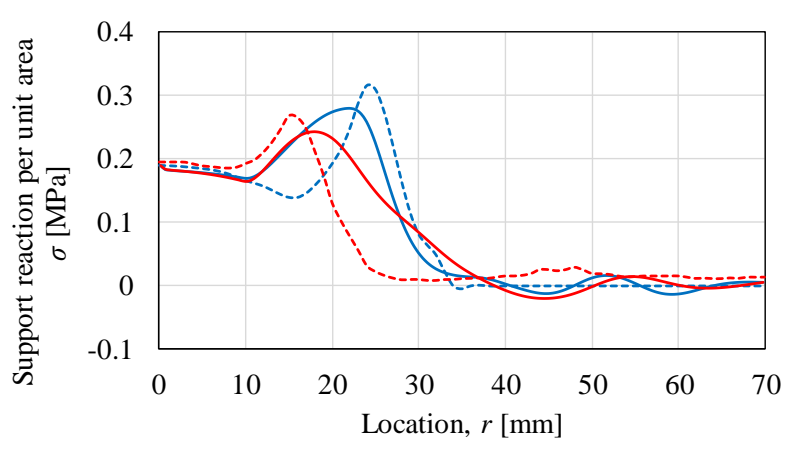

---- Bottom face fixed, Exp. $\quad-$ Bottom face fixed, Est.
----Peripherally clamped, Exp. - Peripherally clamped, Est.

Fig. 3 Distribution of support reaction

the experimental values. Moreover, the peak of the support reaction appears at $r=25 \mathrm{~mm}$ in the bottom face fixed panel and at $r=15 \mathrm{~mm}$ in the peripherally clamped panel. The proposed method can estimate this difference.

Conclusion. The proposed method can estimate the support reaction of the honeycomb core sandwich panel regardless of the fixing method of the panel.

\section{References.}

(1) Kishimoto, Y., Trans. JSME A, 78, (2012), 311-323.

(2) Kishimoto, Y., Mechanical Engineering Journal, 3, (2016), DOI: 10.1299/mej.15-00597. 